\title{
On the ranks of certain ideals of monotone contractions
}

\author{
Leyla Bugay (i) \\ Department of Mathematics, Çukurova University, Adana, 01330, Turkey
}

\begin{abstract}
Let $T_{n}$ be the (full) transformation semigroup, and let $O C T_{n}$ and $O R C T_{n}$ be its subsemigroups of isotone contractions and of monotone contractions on a finite chain $X_{n}=$ $\{1, \ldots, n\}$ under its natural order, respectively. In this study, we obtain the ranks of the ideals $O C T_{n, r}=\left\{\alpha \in O C T_{n}:|\operatorname{im}(\alpha)| \leq r\right\}$ and $O R C T_{n, r}=\left\{\alpha \in O R C T_{n}:|\operatorname{im}(\alpha)| \leq r\right\}$ for $1 \leq r \leq n-1$.
\end{abstract}

Mathematics Subject Classification (2010). 20M20

Keywords. isotone/antitone/monotone transformation, contraction, rank

\section{Introduction}

For $n \in \mathbb{Z}^{+}$let $X_{n}=\{1, \ldots, n\}$ be a finite chain, under its natural order, and let $T_{n}$ be the (full) transformation semigroup, the semigroup of all (full) transformations on $X_{n}$ with usual composition. It is well known that every finite semigroup is embeddable in a transformation semigroup $T_{n}$ for any appropriate $n$, which is correspond to Cayley's theorem for finite symmetric group $S_{n}$, the group of all permutations on $X_{n}$. Hence, the studies on transformation semigroups and their subsemigroups have certain important roles for semigroup theory like as the studies on symmetric groups for group theory.

An element $\alpha \in T_{n}$ is said to be isotone or order-preserving (antitone or order-reversing ) if $x \leq y \Rightarrow x \alpha \leq y \alpha(x \leq y \Rightarrow x \alpha \geq y \alpha)$ for all $x, y \in X_{n}$, and said to be monotone if $\alpha$ is isotone or antitone. Notice that if $|\operatorname{im}(\alpha)|=1$ then $\alpha$ is both isotone and antitone, and so monotone. It is easy to see that the product of two isotone transformations and also the product of two antitone transformations is isotone; and the product of any isotone transformation with any antitone transformation (in each order) is antitone. Then, as known, the subsets

$$
\begin{aligned}
O_{n} & =\left\{\alpha \in T_{n}: \alpha \text { is an isotone transformation on } X_{n}\right\} \text { and } \\
O R_{n} & =\left\{\alpha \in T_{n}: \alpha \text { is a monotone transformation on } X_{n}\right\}
\end{aligned}
$$

are subsemigroups of $T_{n}$. An other notable element in $T_{n}$ is contraction. An element $\alpha \in T_{n}$ is called a contraction if $|x \alpha-y \alpha| \leq|x-y|$ for all $x, y \in X_{n}$, and also the subset

$$
C T_{n}=\left\{\alpha \in T_{n}: \alpha \text { is a contraction on } X_{n}\right\}
$$

Email address: ltanguler@cu.edu.tr

Received: 09.08.2019; Accepted: 13.03.2020 
is a subsemigroup of $T_{n}$. Furthermore, let $O C T_{n}$ and $O R C T_{n}$ be the subsemigroups of $X_{n}$, consists of all isotone contractions and of all monotone contractions, respectively, that is

$$
O C T_{n}=O_{n} \cap C T_{n} \text { and } O R C T_{n}=O R_{n} \cap C T_{n}
$$

for $1 \leq r \leq n$, let

$$
\begin{aligned}
O C T_{n, r} & =\left\{\alpha \in O C T_{n}:|\operatorname{im}(\alpha)| \leq r\right\} \text { and } \\
O R C T_{n, r} & =\left\{\alpha \in O R C T_{n}:|\operatorname{im}(\alpha)| \leq r\right\}
\end{aligned}
$$

which are clearly subsemigroups, even ideals, of $O C T_{n}$ and of $O R C T_{n}$, respectively. Also, we have $O C T_{n, r} \leq O R C T_{n, r}$ for $1 \leq r \leq n$.

For any non-empty subset $U$ of any semigroup $S$, the subsemigroup generated by $U$ is defined as the smallest subsemigroup of $S$ containing $U$ and denoted by $\langle U\rangle$. Moreover, if $S=\langle U\rangle$ then $U$ is said to be a generating set of $S$, and $S$ is said to be the semigroup generated by $U$. Also, the rank of a finitely generated semigroup $S$, a semigroup generated by some finite subsets, is defined by

$$
\operatorname{rank}(S)=\min \{|U|:\langle U\rangle=S\},
$$

and any generating set of $S$ with cardinality $\operatorname{rank}(S)$ is called a minimal generating set of $S$.

As stated in [7], although the notion "contraction" first appeared in [10], algebraic and combinatorial properties of the semigroups $C T_{n}$ and $O C T_{n}$ were investigated first by Adeshola in [1]. Then Adeshola and Umar investigated the cardinalities of some equivalences on $O C T_{n}$ and $O R C T_{n}$ in [2]; and Garba, Ibrahim and Imam presented characterizations of Green's relations on $C T_{n}$ and starred Green's relations on both $C T_{n}$ and $O C T_{n}$ in [7]. Ibrahim, Imam, Adeshola and Bakare investigate the local and global $U$-depth for any generating set $U$ of $O C T_{n}$ as well as the status of $O C T_{n}$ in [14]. An other interesting lack for these semigroups is their ranks. To find the rank of an arbitrary semigroup is an important problem in semigroup theory, similar to find the dimension of an arbitrary group in group theory. Howie, with various co-authors, wrote a lot of studies on ranks of semigroups, see $[8,9,11,13]$ for examples. Then, this problem has gained importance for other researchers working in this field and then many more papers on ranks of semigroups have been written, see $[3,5,6,17]$ for examples. Recently, Toker showed that $\operatorname{rank}\left(O C T_{n, n-1}\right)=n-1$, and $\operatorname{rank}\left(O C T_{n}\right)=n$ for $n \geq 3$, and that

$$
\operatorname{rank}\left(O R C T_{n}\right)= \begin{cases}\frac{n+1}{2} & \text { if } n \text { is an odd number } \\ \frac{n+2}{2} & \text { if } n \text { is an even number }\end{cases}
$$

for $n \geq 1$ in [15]. Now, we examine the ideals $O C T_{n, r}$ and $O R C T_{n, r}$ for $1 \leq r \leq n-1$. Although the part of the motivation of this paper is to state a useful method for finding minimal generating sets of the implied ideals, the main motivation of this paper is to calculate their ranks. Finally, we show that

$$
\operatorname{rank}\left(O C T_{n, r}\right)=\operatorname{rank}\left(O R C T_{n, r}\right)= \begin{cases}n & \text { for } r=1 \\
\left(\begin{array}{l}
n-1 \\
r-1
\end{array}\right) & \text { for } 2 \leq r \leq n-1\end{cases}
$$

in this study.

\section{Preliminaries}

For any $\alpha \in T_{n}$ the height and the kernel of $\alpha$ are defined by

$$
\begin{aligned}
\mathrm{h}(\alpha) & =|\operatorname{im}(\alpha)| \text { and } \\
\operatorname{ker}(\alpha) & =\left\{(x, y): x, y \in X_{n} \text { and } x \alpha=y \alpha\right\},
\end{aligned}
$$


respectively. It is well known that $\operatorname{ker}(\alpha)$ is an equivalence relation on $X_{n}$, and that the set of the equivalence classes obtained by $\operatorname{ker}(\alpha)$, say

$$
X_{n} / \operatorname{ker}(\alpha)=\left\{y \alpha^{-1}: y \in \operatorname{im}(\alpha)\right\},
$$

is a partition of $X_{n}$, called the kernel partition of $\alpha$ and denoted by $\operatorname{kp}(\alpha)$. For $\alpha, \beta \in T_{n}$ it is clear that

$$
\operatorname{kp}(\alpha)=\operatorname{kp}(\beta) \Leftrightarrow \operatorname{ker}(\alpha)=\operatorname{ker}(\beta) .
$$

Let $P=\left\{I_{1}, \ldots, I_{p}\right\}$ be a partition of $X_{n}$ for any $1 \leq p \leq n$. Then we write $I_{i}<I_{j}$ if $x<y$ for all $x \in I_{i}$ and for all $y \in I_{j}$ for $1 \leq i, j \leq p$. Without loss of generality, if $P=\left\{I_{1}<\cdots<I_{p}\right\}$ then $P$ is called an ordered partition. Moreover, a subset $\left\{a_{1}, \ldots, a_{p}\right\}$ of $X_{n}$ is called a representative set (or a transversal or a cross-section) of $P$ if $\mid\left\{a_{1}, \ldots, a_{p}\right\} \cap$ $I_{i} \mid=1$ for each $1 \leq i \leq p$. Also, a subset $\emptyset \neq C \subseteq X_{n}$ is called a convex subset if

$$
x, y \in C \text { and } x \leq z \leq y \Rightarrow z \in C .
$$

Now let $\alpha \in O R C T_{n}=O R_{n} \cap C T_{n}$ with $\mathrm{h}(\alpha)=p(1 \leq p \leq n)$. Since $\alpha \in O R_{n}$, it is well known that the kernel classes of $\alpha$ are convex ordered subsets of $X_{n}$ (see [6, p187] for example), that is there exist $x_{1}, \ldots, x_{p-1} \in X_{n}$ such that the kernel classes of $\alpha$ are $I_{i}=\left\{x_{i-1}+1, \ldots, x_{i}\right\}$ for $1 \leq i \leq p$ where $x_{0}=0$ and $x_{p}=n$, and so the kernel partition of $\alpha \in O R_{n}$ is $\operatorname{kp}(\alpha)=P=\left\{I_{1}<\cdots<I_{p}\right\}$. Moreover, since $\alpha \in C T_{n}$ it is also well known that $\operatorname{im}(\alpha)$ is a convex subset of $X_{n}$ (see [2, Lemma 1.2] for example), that is there exist $a \in X_{n}$ such that $\operatorname{im}(\alpha)=A=\{a, a+1, \ldots, a+p-1\}$. Then $\alpha$ has the following tabular form:

$$
\alpha=\left(\begin{array}{cccc}
I_{1} & I_{2} & \cdots & I_{p} \\
a & a+1 & \cdots & a+p-1
\end{array}\right), \text { or shortly } \alpha=\left(\begin{array}{c}
P \\
A
\end{array}\right)
$$

if $\alpha$ is isotone, and

$$
\alpha=\left(\begin{array}{cccc}
I_{1} & I_{2} & \cdots & I_{p} \\
a+p-1 & a+p-2 & \cdots & a
\end{array}\right) \text { or shortly } \alpha=\left(\begin{array}{c}
P \\
A^{R}
\end{array}\right)
$$

if $\alpha$ is antitone. For any $1 \leq p \leq n$, there exist $\left(\begin{array}{c}n-1 \\ p-1\end{array}\right)$ many convex ordered partition of $X_{n}$ into $p$ subsets, and $n-p+1$ many convex subset of $X_{n}$ with $p$ elements. Therefore, it is easy to see that $\left|O C T_{n, 1}\right|=\left|O R C T_{n, 1}\right|=n$ and that

$$
\begin{gathered}
\left|O C T_{n, r}\right|=\sum_{p=1}^{r}\left(\begin{array}{l}
n-1 \\
p-1
\end{array}\right)(n-p+1) \text { and } \\
\left|O R C T_{n, r}\right|=n+2 \sum_{p=2}^{r}\left(\begin{array}{l}
n-1 \\
p-1
\end{array}\right)(n-p+1)
\end{gathered}
$$

for $2 \leq r \leq n-1$.

As usual, an element $x$ in any semigroup $S$ is called an idempotent if $x^{2}=x$, and the set of all idempotents in any subset $\emptyset \neq U \subseteq S$ denoted by $E(U)$. Then it is well known that

In particular,

$$
\alpha \in E\left(T_{n}\right) \Leftrightarrow \operatorname{im}(\alpha)=\operatorname{fix}(\alpha)=\left\{x \in X_{n}: x \alpha=x\right\} .
$$

$\alpha \in E\left(O C T_{n}\right) \Leftrightarrow \operatorname{im}(\alpha)$ is a representative set of $\mathrm{kp}(\alpha)$,

and clearly for any $\alpha \in O C T_{n}, \operatorname{im}(\alpha)=\{a, a+1, \ldots, a+p-1\}(1 \leq p \leq n)$ is a representative set of $\operatorname{kp}(\alpha)$ if and only if

$$
\operatorname{kp}(\alpha)=\{\{1, \ldots, a\},\{a+1\}, \ldots,\{a+p-2\},\{a+p-1, \ldots, n\}\} .
$$

Notice that, for each convex subset $\emptyset \neq A \subseteq X_{n}$ there exists a unique convex ordered partition $P$ of $X_{n}$ such that $A$ is a representative set of $P$, and so there exists a unique 
idempotent in $O C T_{n}$ such that $\operatorname{im}(\alpha)=A$. Thus, since there exist $n-p+1$ many convex subsets of $X_{n}$ with cardinality $p$ and since each idempotent have to be isotone, it follows that

$$
\left|E\left(O C T_{n, r}\right)\right|=\left|E\left(O R C T_{n, r}\right)\right|=\sum_{p=1}^{r}(n-p+1)
$$

and so

$$
\left|E\left(O C T_{n}\right)\right|=\left|E\left(O R C T_{n}\right)\right|=\sum_{p=1}^{n}(n-p+1)=\frac{n(n+1)}{2}
$$

which is first appeared in [2, Corollary 2.14].

After stating some combinatorial results in $O C T_{n, r}$ and $O R C T_{n, r}$, now we give some properties about their algebraic structures. In general, one of the most common interest on algebraic structure of any semigroup $S$ is to examine the characterization of the Green's equivalences $\mathcal{L}, \mathcal{R}, \mathcal{J}, \mathcal{D}, \mathcal{H}$ on $S$, especially if a semigroup $S$ is regular (for the definitions and certain properties of Green's equivalences and for the other undefined terms in semigroup theory see $[4,12]$ for examples). For non-regular semigroups, it is more common to examine the characterization of the starred Green's equivalences $\mathcal{L}^{*}, \mathcal{R}^{*}, \mathcal{J}^{*}, \mathcal{H}^{*}, \mathcal{D}^{*}$, the generalization of Green's equivalences. On any semigroup $S$, the starred Green's equivalence $\mathcal{L}^{*}\left(\mathcal{R}^{*}\right)$ is defined for $a, b \in S$ by the rule that $a \mathcal{L}^{*} b\left(a \mathcal{R}^{*} b\right)$ if and only if $a \mathcal{L} b$ $(a \mathcal{R} b)$ on some over-semigroup of $S$ (a semigroup containing $S$ as a subsemigroup). These equivalences also have the following characterizations:

$$
\begin{aligned}
& a \mathcal{L}^{*} b \Leftrightarrow a x=a y \text { if and only if } b x=b y \text { for all } x, y \in S^{1} \\
& a \mathcal{R}^{*} b \Leftrightarrow x a=y a \text { if and only if } x b=y b \text { for all } x, y \in S^{1}
\end{aligned}
$$

for $a, b \in S$ where $S^{1}$ is the monoid obtained from $S$ by adjoining an identity if necessary. Moreover, the equivalences $\mathcal{H}^{*}$ and $\mathcal{D}^{*}$ are defined as the intersection and the join of the equivalences $\mathcal{L}^{*}$ and $\mathcal{R}^{*}$, respectively, say $\mathcal{H}^{*}=\mathcal{L}^{*} \cap \mathcal{R}^{*}$ and $\mathcal{D}^{*}=\mathcal{L}^{*} \vee \mathcal{R}^{*}$, and it is well known that $\mathcal{D}^{*}=\mathcal{L}^{*} \circ \mathcal{R}^{*}$.

Let $S \in\left\{O C T_{n, r}, O R C T_{n, r}\right\}$ for $1 \leq r \leq n-1$ unless otherwise stated. For $\alpha, \beta \in S$, it is a routine matter to prove, as in [7, Theorem 4.1], that

(i) $\alpha \mathcal{L}^{*} \beta \Leftrightarrow \operatorname{im}(\alpha)=\operatorname{im}(\beta)$,

(ii) $\alpha \mathcal{R}^{*} \beta \Leftrightarrow \operatorname{ker}(\alpha)=\operatorname{ker}(\beta)$,

(iii) $\alpha \mathcal{H}^{*} \beta \Leftrightarrow \operatorname{ker}(\alpha)=\operatorname{ker}(\beta)$ and $\operatorname{im}(\alpha)=\operatorname{im}(\beta)$, and

(iv) $\alpha \mathcal{D}^{*} \beta \Leftrightarrow|\operatorname{im}(\alpha)|=|\operatorname{im}(\beta)|$,

and also $\mathcal{D}^{*}=\mathcal{J}^{*}$. Then, for $1 \leq p \leq r \leq n-1$, we denote the starred Green's $\mathcal{D}^{*}$-class of all elements in $S$ of height $p$ by $D_{p}^{*}$, that is

$$
D_{p}^{*}=\{\alpha \in S:|\operatorname{im}(\alpha)|=p\} .
$$

It is clear that there exist $r$ many $\mathcal{D}^{*}$-classes, namely $D_{1}^{*}, \ldots, D_{r}^{*}$, and $S$ is the disjoint union of $D_{1}^{*}, \ldots, D_{r}^{*}$. Moreover, there exist $n-p+1$ many $\mathcal{L}^{*}$-classes and $\left(\begin{array}{c}n-1 \\ p-1\end{array}\right)$ many $\mathcal{R}^{*}$-classes in $D_{p}^{*}$ for each $1 \leq p \leq r$. As also shown in [15] that, it is a routine matter to show that $\mathcal{D}_{p}^{*} \subseteq\left\langle\mathcal{D}_{p+1}^{*}\right\rangle$, more explicitly, for any $\alpha \in \mathcal{D}_{p}^{*}$ there exist $\beta, \gamma \in \mathcal{D}_{p+1}^{*}$ such that $\alpha=\beta \gamma$, for any $1 \leq p \leq n-2$. Therefore, for any subset $\emptyset \neq U \subseteq S$,

$$
S=\langle U\rangle \Leftrightarrow \mathcal{D}_{r}^{*} \subseteq\langle U\rangle .
$$

Also, it is clear that $\mathcal{D}_{r}^{*}$ can be generated only by its own elements. Hence, it is enough to examine only the non-empty subsets of $\mathcal{D}_{r}^{*}$ to find a (minimal) generating set of $S$.

Next we give a lemma which is useful for this manuscript. 
Lemma 2.1. For $1 \leq p \leq n-1$ and $2 \leq k$ let $\alpha_{1}, \ldots, \alpha_{k} \in D_{p}^{*}$ in $S$ where $S \in$ $\left\{O C T_{n, r}, O R C T_{n, r}\right\}$ for $1 \leq r \leq n-1$. Then

$$
\begin{aligned}
\alpha_{1} \cdots \alpha_{k} \in D_{p}^{*} & \Leftrightarrow \alpha_{i} \alpha_{i+1} \in D_{p}^{*} \text { for each } 1 \leq i \leq k-1 \\
& \Leftrightarrow \operatorname{im}\left(\alpha_{i}\right) \text { is a representative set of } \operatorname{kp}\left(\alpha_{i+1}\right) \text { for each } 1 \leq i \leq k-1 .
\end{aligned}
$$

Proof. The proof is similar to the proof of [3, Lemma 2].

Now we give some definitions about digraphs. A digraph (directed graph) is an ordered pair $\Pi=(V, E)$ where $V$ is a set whose elements are called vertices and $E \subseteq V \times V$ is a set of ordered pairs whose elements are called arrows or directed edges. For some elements $u_{1}, \ldots, u_{k} \in V(k \geq 2)$ that do not need to be different if $\left(u_{1}, u_{2}\right),\left(u_{2}, u_{3}\right), \ldots,\left(u_{k-1}, u_{k}\right) \in$ $E$, then $u_{1} \rightarrow u_{2} \rightarrow \cdots \rightarrow u_{k}$ is called a walk from $u_{1}$ to $u_{k}$. In particular, for distinct vertices $u_{1}, \ldots, u_{k} \in V$ where $k \geq 1$, the closed walk $u_{1} \rightarrow \cdots \rightarrow u_{k} \rightarrow u_{1}$ is called a cycle. For any walk $u_{1} \rightarrow \cdots \rightarrow u_{k}(2 \leq k)$, the ordered product $u_{1} u_{2} \cdots u_{k}$ is called a consecutive product. (For unexplained terms about digraphs, see [16] for example.)

Finally, we define a new digraph $\mathfrak{D}_{U}$ for any $\emptyset \neq U \subseteq D_{r}^{*}$ which will be used in the main theorem of this manuscript. Let $\emptyset \neq U \subseteq D_{r}^{*}$ in $S$ where $S \in\left\{O C T_{n, r}, O R C T_{n, r}\right\}$ for $1 \leq r \leq n-1$. Then the digraph $\mathfrak{D}_{U}=(U, E)$ is defined by

$$
\begin{aligned}
E & =\left\{(\alpha, \beta): \alpha \beta \in D_{r}^{*}\right\} \\
& =\{(\alpha, \beta): \operatorname{im}(\alpha) \text { is a representative set of } \operatorname{kp}(\beta)\} .
\end{aligned}
$$

Notice that, for any $\alpha \in O R C T_{n, r}, \operatorname{im}(\alpha)=\{a, a+1, \ldots, a+r-1\}$ is a representative set of $\operatorname{kp}(\beta)$ if and only if $\operatorname{kp}(\beta)=\{\{1, \ldots, a\},\{a+1\}, \ldots,\{a+r-2\},\{a+r-1, \ldots, n\}\}$.

\section{Rank of $O C T_{n, r}$}

Theorem 3.1. Let $1 \leq r \leq n-1$ and let $\emptyset \neq U \subseteq D_{r}^{*}$. Then $U$ is a generating set of $O C T_{n, r}$ if and only if, for each convex ordered partition $P$ of $X_{n}$ into $r$ subsets and for each convex subset $A$ of $X_{n}$ with cardinality $r$, there exist $\alpha, \beta \in U$ such that

(i) $\mathrm{kp}(\alpha)=P$,

(ii) $\operatorname{im}(\beta)=A$ and

(iii) $\alpha=\beta$ or there exists a walk from $\alpha$ to $\beta$ in the digraph $\mathfrak{D}_{U}$.

Proof. $(\Rightarrow)$ For any convex ordered partition $P$ of $X_{n}$ into $r$ subsets and any convex subset $A$ of $X_{n}$ with cardinality $r(1 \leq r \leq n-1)$, consider the unique isotone contraction $\gamma \in D_{r}^{*}$ with kernel partition $P$ and image set $A$. If $\gamma \in U$, then the result is clear. Now let $\gamma \in D_{r}^{*} \backslash U$. Since $\emptyset \neq U \subseteq D_{r}^{*}$ is a generating set of $O C T_{n, r}$, then there exist $\alpha_{1}, \ldots, \alpha_{m} \in$ $U$ such that $\alpha_{1} \cdots \alpha_{m}=\gamma$ for $m \geq 2$. Then we have $\operatorname{ker}\left(\alpha_{1}\right) \subseteq \operatorname{ker}(\gamma), \operatorname{im}(\gamma) \subseteq \operatorname{im}\left(\alpha_{m}\right)$ and $\alpha_{1}, \ldots, \alpha_{m}, \gamma \in D_{r}^{*}$, and so $\operatorname{kp}\left(\alpha_{1}\right)=\operatorname{kp}(\gamma)=P$ and $\operatorname{im}\left(\alpha_{m}\right)=\operatorname{im}(\gamma)=A$. Thus the first two conditions are satisfied. From Lemma 2.1, also we have $\alpha_{i} \alpha_{i+1} \in D_{r}^{*}$ for each $1 \leq i \leq m-1$, and so $\alpha_{1} \rightarrow \cdots \rightarrow \alpha_{m}$ is a walk from $\alpha_{1}$ to $\alpha_{m}$ in $\mathfrak{D}_{U}$.

$(\Leftarrow)$ Let $\gamma \in D_{r}^{*}$ and let $\operatorname{kp}(\gamma)=P$ and $\operatorname{im}(\gamma)=A$. From the assumptions, there exist $\alpha, \beta \in U$ such that $\operatorname{kp}(\alpha)=P=\operatorname{kp}(\gamma), \operatorname{im}(\beta)=A=\operatorname{im}(\gamma)$, and $\alpha=\beta$ or there exists a walk from $\alpha$ to $\beta$ in $\mathfrak{D}_{U}$. If $\alpha=\beta$ clearly $\alpha=\beta=\gamma \in U$, otherwise, let $\alpha=\alpha_{1} \rightarrow \cdots \rightarrow \alpha_{m}=\beta(m \geq 2)$ be a walk from $\alpha$ to $\beta$ in $\mathfrak{D}_{U}$, and let $\xi$ be the consecutive product of all elements on this walk, say $\xi=\alpha_{1} \cdots \alpha_{m}$. Similarly, we have $\operatorname{ker}(\alpha) \subseteq \operatorname{ker}(\xi)$ and $\operatorname{im}(\xi) \subseteq \operatorname{im}(\beta)$. Moreover, it follows from the definition of the digraph $\mathfrak{D}_{U}$ and Lemma 2.1 that $\xi \in D_{r}^{*}$, and so $\operatorname{kp}(\xi)=\operatorname{kp}(\alpha)=\operatorname{kp}(\gamma)$ and $\operatorname{im}(\xi)=\operatorname{im}(\beta)=\operatorname{im}(\gamma)$. Hence, $\gamma=\xi \in\langle U\rangle$, and so $D_{r}^{*} \subseteq\langle U\rangle$, as required.

In conclusion, if $O C T_{n, r}=\langle U\rangle$ for any $\emptyset \neq U \subseteq \mathcal{D}_{r}^{*}$, then for each $\alpha \in \mathcal{D}_{r}^{*}$ there exists at least one element in $U$ which is $\mathcal{L}^{*}$-equivalent to $\alpha$ and there exists at least one element in $U$ which is $\mathcal{R}^{*}$-equivalent to $\alpha$. Thus $U$ must cover the $\mathcal{L}^{*}$-classes and also the $\mathcal{R}^{*}$-classes in $D_{r}^{*}$. Hence $\operatorname{rank}\left(O C T_{n, 1}\right) \geq n$ and $\operatorname{rank}\left(O C T_{n, r}\right) \geq\left(\begin{array}{l}n-1 \\ r-1\end{array}\right)$ for $2 \leq r \leq n-1$. 
Corollary 3.2. $O C T_{n, 1}=\left\{\left(\begin{array}{c}X_{n} \\ \{1\}\end{array}\right), \ldots,\left(\begin{array}{c}X_{n} \\ \{n\}\end{array}\right)\right\}$ and there is no proper generating set of $O C T_{n, 1}$.

Lemma 3.3. For $2 \leq r \leq n-1$, let $m=n-r+1$ and let $I_{1}, \ldots, I_{m}$ be all of the convex subsets of $X_{n}$ with cardinality $r$. Then there exist $m$ different convex ordered partitions $P_{1}, \ldots, P_{m}$ of $X_{n}$ into $r$ subsets such that $I_{i}$ is a representative set of $P_{i}$ for each $1 \leq i \leq m$.

Proof. For each $1 \leq i \leq m$, let $I_{i}=\{i, i+1, \ldots, i+r-1\}$ and let $P_{i}=\{\{1, \ldots, i\},\{i+$ $1\}, \ldots,\{i+r-2\},\{i+r-1, \ldots, n\}\}$. Then the result is clear.

Lemma 3.4. For $2 \leq r \leq n-1$, let $m=n-r+1$, and let $I_{1}, \ldots, I_{m}$ be all of the convex subsets of $X_{n}$ with cardinality $r$. Then there exist $\alpha_{1}, \ldots, \alpha_{m} \in D_{r}^{*}$ in $O C T_{n, r}$ such that

(i) $\mathrm{kp}\left(\alpha_{i}\right) \neq \mathrm{kp}\left(\alpha_{j}\right)$ if $1 \leq i \neq j \leq m$ and

(ii) $\operatorname{im}\left(\alpha_{i}\right)=I_{i}$ is a representative set of $\mathrm{kp}\left(\alpha_{i+1}\right)$, for each $1 \leq i \leq m$ where $\alpha_{m+1}=$ $\alpha_{1}$.

Proof. Let $m=n-r+1$, and let $I_{1}, \ldots, I_{m}$ be all of the convex subsets of $X_{n}$ with cardinality $r$ for $2 \leq r \leq n-1$. Then it follows from Lemma 3.3 that there exist $m$ different convex ordered partitions $P_{1}, \ldots, P_{m}$ of $X_{n}$ into $r$ subsets such that $I_{i}$ is a representative set of $P_{i}$ for each $1 \leq i \leq m$. Without loss of generality, let $\alpha_{i} \in D_{r}^{*}$ be the unique isotone contraction represented by $\operatorname{kp}\left(\alpha_{i}\right)=P_{i-1}$ and $\operatorname{im}\left(\alpha_{i}\right)=I_{i}$ for each $1<i \leq m$ where $P_{0}=P_{m}$. Then the result is clear.

For $2 \leq r \leq n-1$, let $I_{1}, \ldots, I_{m}$ be all of the convex subsets of $X_{n}$ with cardinality $r$, and let $R_{1}^{*}, \ldots, R_{t}^{*}$ be all of the starred Green's $\mathcal{R}^{*}$-classes in $D_{r}^{*}$ where $m=n-r+1$ and $t=\left(\begin{array}{l}n-1 \\ r-1\end{array}\right)$. It follows from Lemma 3.4 that there exist $\alpha_{1}, \ldots, \alpha_{m} \in D_{r}^{*}$ in $O C T_{n, r}$ such that

(i) $\operatorname{kp}\left(\alpha_{i}\right) \neq \mathrm{kp}\left(\alpha_{j}\right)$ if $1 \leq i \neq j \leq m$ and

(ii) $\operatorname{im}\left(\alpha_{i}\right)=I_{i}$ is a representative set of $\mathrm{kp}\left(\alpha_{i+1}\right)$, for each $1 \leq i \leq m$ where $\alpha_{m+1}=$ $\alpha_{1}$.

Without loss of generality, we may assume that $\alpha_{i} \in R_{i}^{*}$ for $1 \leq i \leq m$, and take an arbitrary contraction $\alpha_{m+j}$ from $R_{m+j}^{*}$ for each $1 \leq j \leq t-m$. Then consider the set $U=\left\{\alpha_{1}, \ldots, \alpha_{m}, \alpha_{m+1}, \ldots, \alpha_{t}\right\}$ which satisfies the first two conditions of Theorem 3.1. Also, it is easy to see that,

$$
\alpha_{1} \rightarrow \cdots \rightarrow \alpha_{m} \rightarrow \alpha_{1}
$$

is a cycle in $\mathfrak{D}_{U}$ and, for each $1 \leq j \leq t-m$, there exists $1 \leq i \leq m$ such that $\alpha_{m+j} \rightarrow \alpha_{i}$ is a walk in $\mathfrak{D}_{U}$. Therefore, for any $\gamma \in D_{r}^{*} \backslash U$, there exist $\alpha_{k}, \alpha_{l} \in U$ such that $\operatorname{kp}\left(\alpha_{k}\right)=$ $\operatorname{kp}(\gamma), \operatorname{im}\left(\alpha_{l}\right)=\operatorname{im}(\gamma)$, and that there exists a walk from $\alpha_{k}$ to $\alpha_{l}$ in $\mathfrak{D}_{U}$. Indeed to generate $\gamma \in D_{r}^{*} \backslash U$, we can use the consecutive product of all isotone contractions on a suitable walk from the vertex $\alpha_{k}$ to the vertex $\alpha_{l}$. Then it follows from Theorem 3.1 that $U$ is a generating set of $O C T_{n, r}$, and so we have the following theorem.

\section{Theorem 3.5.}

$$
\operatorname{rank}\left(O C T_{n, r}\right)=\left\{\begin{array}{ll}
n & \text { for } r=1 \\
\left(\begin{array}{l}
n-1 \\
r-1
\end{array}\right) & \text { for } 2 \leq r \leq n-1
\end{array} .\right.
$$

Proof. The result follows from Corollary 3.2 and the fact $\operatorname{rank}\left(O C T_{n, r}\right) \geq\left(\begin{array}{l}n-1 \\ r-1\end{array}\right)$ for $2 \leq r \leq n-1$. 


\section{Rank of $O R C T_{n, r}$}

Notice that $O C T_{n, 1}=O R C T_{n, 1}$. Therefore, unless otherwise stated, in this section we consider the case $2 \leq r \leq n-1$. Also, recall that for any subset $\emptyset \neq U \subseteq O R C T_{n, r}, U$ is a generating set of $O R C T_{n, r}$ if and only if $\mathcal{D}_{r}^{*} \subseteq\langle U\rangle$.

Lemma 4.1. $O R C T_{n, r}=\left\langle R\left(D_{r}^{*}\right)\right\rangle$ where $R\left(D_{r}^{*}\right)=\left\{\alpha \in D_{r}^{*}: \alpha\right.$ is antitone $\}$.

Proof. Let $\alpha \in D_{r}^{*}$ be a isotone contraction with the following tabular form:

$$
\alpha=\left(\begin{array}{cccc}
A_{1} & A_{2} & \cdots & A_{r} \\
a & a+1 & \cdots & a+r-1
\end{array}\right) .
$$

Then clearly we have $\alpha=\beta \gamma$ where

$$
\begin{gathered}
\beta=\left(\begin{array}{cccc}
A_{1} & A_{2} & \cdots & A_{r} \\
a+r-1 & a+r-2 & \cdots & a
\end{array}\right) \in R\left(D_{r}^{*}\right) \text { and } \\
\gamma=\left(\begin{array}{ccccc}
\{1, \ldots, a\} & \{a+1\} & \cdots & \{a+r-2\} & \{a+r-1, \ldots, n\} \\
a+r-1 & a+r-2 & \cdots & a+1 & a
\end{array}\right) \in R\left(D_{r}^{*}\right) .
\end{gathered}
$$

Thus, $D_{r}^{*} \subseteq\left\langle R\left(D_{r}^{*}\right)\right\rangle$, and so $O R C T_{n, r}=\left\langle R\left(D_{r}^{*}\right)\right\rangle$.

As a result, $\emptyset \neq U \subseteq D_{r}^{*}$ is a generating set of $O R C T_{n, r}$ if and only if $R\left(D_{r}^{*}\right) \subseteq\langle U\rangle$.

Theorem 4.2. For $2 \leq r \leq n-1, \emptyset \neq U \subseteq D_{r}^{*}$ is a generating set of $O R C T_{n, r}$ if and only if, for each convex ordered partition $P$ of $X_{n}$ into $r$ subsets and for each convex subset $A$ of $X_{n}$ with cardinality $r$, there exist $\alpha, \beta \in U$ such that

(i) $\operatorname{kp}(\alpha)=P$,

(ii) $\operatorname{im}(\beta)=A$, and

(iii) $\alpha=\beta \in R\left(D_{r}^{*}\right)$ or there exists a walk from $\alpha$ to $\beta$ in the digraph $\mathfrak{D}_{U}$ such that the number of vertex on the walk which is antitone is an odd number.

Proof. $(\Rightarrow)$ For any convex ordered partition $P$ of $X_{n}$ into $r$ subsets and any convex subset $A$ of $X_{n}$ with cardinality $r(2 \leq r \leq n-1)$, consider the unique antitone contraction $\gamma \in R\left(D_{r}^{*}\right)$ with kernel partition $P$ and image set $A$. If $\gamma \in U$, then the result is clear. Now let $\gamma \in R\left(D_{r}^{*}\right) \backslash U$. Since $\emptyset \neq U \subseteq D_{r}^{*}$ is a generating set of $O R C T_{n, r}$, then $R\left(D_{r}^{*}\right) \subseteq\langle U\rangle$, and so there exist $\alpha_{1}, \ldots, \alpha_{m} \in U$ such that $\alpha_{1} \cdots \alpha_{m}=\gamma$ for $m \geq 2$. Then we have $\operatorname{ker}\left(\alpha_{1}\right) \subseteq \operatorname{ker}(\gamma), \operatorname{im}(\gamma) \subseteq \operatorname{im}\left(\alpha_{m}\right)$ and $\alpha_{1}, \ldots, \alpha_{m}, \gamma \in D_{r}^{*}$, and so $\operatorname{kp}\left(\alpha_{1}\right)=\operatorname{kp}(\gamma)=P$ and $\operatorname{im}\left(\alpha_{m}\right)=\operatorname{im}(\gamma)=A$. Thus the first two conditions are satisfied. Moreover, we have $\alpha_{i} \alpha_{i+1} \in D_{r}^{*}$ for each $1 \leq i \leq m-1$ from Lemma 2.1 , and so $\alpha_{1} \rightarrow \cdots \rightarrow \alpha_{m}$ is a walk in the digraph $\mathfrak{D}_{U}$. Moreover, since $\gamma \in R\left(D_{r}^{*}\right)$, it is easy to see that the number of vertex on the walk which is antitone has to be an odd number.

$(\Leftarrow)$ Let $\gamma \in R\left(D_{r}^{*}\right)$ and let $\operatorname{kp}(\gamma)=P$ and $\operatorname{im}(\gamma)=A$. Then from the assumptions, there exist $\alpha, \beta \in U$ such that $\operatorname{kp}(\alpha)=P=\operatorname{kp}(\gamma), \operatorname{im}(\beta)=A=\operatorname{im}(\gamma)$, and $\alpha=\beta \in$ $R\left(D_{r}^{*}\right)$ or there exists a walk from $\alpha$ to $\beta$ in the digraph $\mathfrak{D}_{U}$ such that the number of vertex on the walk which is antitone is an odd number. If $\alpha=\beta \in R\left(D_{r}^{*}\right)$ clearly $\alpha=\beta=\gamma \in U$, otherwise, let $\xi$ be the consecutive product of all elements on the walk. Then, as in the proof of Theorem 3.1, $\mathrm{kp}(\xi)=\mathrm{kp}(\alpha)=\mathrm{kp}(\gamma)$ and $\operatorname{im}(\xi)=\operatorname{im}(\beta)=\operatorname{im}(\gamma)$. Moreover, since the number of vertex on the walk which is antitone is an odd number, we have $\xi \in R\left(D_{r}^{*}\right)$. Thus, $\gamma=\xi \in\langle U\rangle$, and so $R\left(D_{r}^{*}\right) \subseteq\langle U\rangle$, as required.

Lemma 4.3. For $2 \leq r \leq n-1$ let $m=n-r+1$ and let $I_{1}, \ldots, I_{m}$ be all of the convex subsets of $X_{n}$ with cardinality $r$. Then there exist $\alpha_{1}, \ldots, \alpha_{m} \in D_{r}^{*}$ in $O R C T_{n, r}$ such that

(i) $\mathrm{kp}\left(\alpha_{i}\right) \neq \mathrm{kp}\left(\alpha_{j}\right)$ if $1 \leq i \neq j \leq m$ and

(ii) $\mathrm{im}\left(\alpha_{i}\right)=I_{i}$ is a representative set of $\mathrm{kp}\left(\alpha_{i+1}\right)$, for each $1 \leq i \leq m$ where $\alpha_{m+1}=$ $\alpha_{1}$. 
Proof. It can be proved as in Lemma 3.4.

Let $I_{1}, \ldots, I_{m}$ be all of the convex subsets of $X_{n}$ with cardinality $r$, and let $R_{1}^{*}, \ldots, R_{t}^{*}$ be all of the starred Green's $\mathcal{R}^{*}$-classes in $D_{r}^{*}$ where $m=n-r+1$ and $t=\left(\begin{array}{c}n-1 \\ r-1\end{array}\right)$. It follows from Lemma 4.3 that there exist $\alpha_{1}, \ldots, \alpha_{m} \in D_{r}^{*}$ in $O R C T_{n, r}$ such that

(i) $\mathrm{kp}\left(\alpha_{i}\right) \neq \mathrm{kp}\left(\alpha_{j}\right)$ if $1 \leq i \neq j \leq m$ and

(ii) $\operatorname{im}\left(\alpha_{i}\right)=I_{i}$ is a representative set of $\mathrm{kp}\left(\alpha_{i+1}\right)$, for each $1 \leq i \leq m$ where $\alpha_{m+1}=$ $\alpha_{1}$.

Without loss of generality, we can take $\alpha_{1}$ as an antitone contraction and $\alpha_{i}$ as an isotone contraction for each $2 \leq i \leq m$. Also, we may assume that $\alpha_{i} \in R_{i}^{*}$ for each $1 \leq i \leq m$, and take an arbitrary isotone contraction $\alpha_{m+j}$ from $R_{m+j}^{*}$ for each $1 \leq j \leq t-m$. Then consider the set $U=\left\{\alpha_{1}, \ldots, \alpha_{m}, \alpha_{m+1}, \ldots, \alpha_{t}\right\}$. It is easy to see that

$$
\alpha_{1} \rightarrow \cdots \rightarrow \alpha_{m} \rightarrow \alpha_{1}
$$

is a cycle in the digraph $\mathfrak{D}_{U}$. Moreover, for each $1 \leq j \leq t-m$, there exists $1 \leq d_{j} \leq m$ such that $\operatorname{im}\left(\alpha_{m+j}\right)=\operatorname{im}\left(\alpha_{d_{j}}\right)$. If $1 \leq d_{j} \leq m-1$ then clearly $\alpha_{m+j} \rightarrow \alpha_{d_{j}+1}$ is a walk in $\mathfrak{D}_{U}$, and if $d_{j}=m$ then clearly $\alpha_{m+j} \rightarrow \alpha_{1}$ is a walk in $\mathfrak{D}_{U}$. Hence, for any convex ordered partition $P$ of $X_{n}$ into $r$ subsets and for any convex subset $A$ of $X_{n}$ with cardinality $r$, there exists a unique $\alpha_{k} \in U$ such that $\operatorname{kp}\left(\alpha_{k}\right)=P$ and there exist unique $\alpha_{l} \in\left\{\alpha_{1}, \ldots, \alpha_{m}\right\}$ such that $\operatorname{im}\left(\alpha_{l}\right)=A$. Therefore, first two conditions of Theorem 4.2 are satisfied. Now we examine the last condition of Theorem 4.2. There exist three cases.

Case 1: When $\alpha_{k}=\alpha_{l}=\alpha_{1}$, the result is clear since $\alpha_{1} \in R\left(D_{r}^{*}\right)$.

Case 2: When $\alpha_{k}=\alpha_{l} \neq \alpha_{1}$, consider the walk

$$
\alpha_{l} \rightarrow \alpha_{l+1} \rightarrow \cdots \rightarrow \alpha_{m} \rightarrow \alpha_{1} \rightarrow \cdots \rightarrow \alpha_{l-1} \rightarrow \alpha_{l},
$$

which contains only one antitone map. Hence, the third condition of Theorem 4.2 is satisfied.

Case 3: When $\alpha_{k} \neq \alpha_{l}$, there exist $p(p \geq 0)$ distinct elements $\beta_{1}, \ldots, \beta_{p} \in U \backslash\left\{\alpha_{k}, \alpha_{l}\right\}$ such that the shortest walk from $\alpha_{k}$ to $\alpha_{l}$ is

$$
\alpha_{k} \rightarrow \beta_{1} \rightarrow \cdots \rightarrow \beta_{p} \rightarrow \alpha_{l} .
$$

If $\alpha_{1} \in\left\{\alpha_{k}, \beta_{1}, \ldots, \beta_{p}, \alpha_{l}\right\}$, then the third condition of Theorem 4.2 is satisfied. If $\alpha_{1} \notin$ $\left\{\alpha_{k}, \beta_{1}, \ldots, \beta_{p}, \alpha_{l}\right\}$ then consider the walk

$$
\alpha_{k} \rightarrow \beta_{1} \rightarrow \cdots \rightarrow \beta_{p} \rightarrow \alpha_{l} \rightarrow \alpha_{l+1} \rightarrow \cdots \rightarrow \alpha_{m} \rightarrow \alpha_{1} \rightarrow \alpha_{2} \rightarrow \cdots \rightarrow \alpha_{l-1} \rightarrow \alpha_{l},
$$

which satisfies the third condition of Theorem 4.2.

Therefore, from Theorem 4.2, $U$ is a generating set of $O R C T_{n, r}$, and so we have the following theorem.

Theorem 4.4. $\operatorname{rank}\left(O R C T_{n, r}\right)=\left\{\begin{array}{ll}n & \text { for } r=1 \\ \left(\begin{array}{l}n-1 \\ r-1\end{array}\right) & \text { for } 2 \leq r \leq n-1\end{array}\right.$.

Proof. The result follows from Corollary 3.2 and the facts $O R C T_{n, 1}=O C T_{n, 1}$ and $\operatorname{rank}\left(O R C T_{n, r}\right) \geq\left(\begin{array}{l}n-1 \\ r-1\end{array}\right)$ for $2 \leq r \leq n-1$.

\section{References}

[1] A.D. Adeshola, Some semigroups of full contraction mappings of a finite chain, PH.D Thesis, University of Ilorin, Nigeria, 2013.

[2] A.D. Adeshola and A. Umar, Combinatorial results for certain semigroups of orderpreserving full contraction mappings of a Finite Chain, J. Combin. Math. Combin. Comput. 106, 37-49, 2018.

[3] H. Ayık and L. Bugay, Generating sets of finite transformation semigroups PK $(n, r)$ and $K(n, r)$. Comm. Algebra, 43, 412-422, 2015. 
[4] O. Ganyushkin and V. Mazorchuk, Classical Finite Transformation Semigroups, Springer-Verlag, Berlin, Germany, 2009.

[5] G.U. Garba, Idempotents in partial transformation semigroups, Proc. Roy. Soc. Edinburgh, 116 (A), 359-366, 1990.

[6] G.U. Garba, On the idempotent ranks of certain semigroups of order-preserving transformations, Port. Math. 51, 185-204, 1994.

[7] G.U. Garba and M.J. Ibrahim and A.T. Imam, On certain semigroups of full contraction maps of a finite chain, Turkish J. Math. 41, 500-507, 2017.

[8] G.M.S. Gomes and J.M. Howie, On the ranks of certain finite semigroups of transformations, Math. Proc. Cambridge Philos. Soc. 101 (3), 395-403, 1987.

[9] G.M.S. Gomes and J.M. Howie, On the ranks of certain semigroups of orderpreserving transformations, Semigroup Forum, 45 (3), 272-282, 1992.

[10] P.M. Higgins J.M. Howie, J.D. Mitchell and N. Ruskuc, Countable versus uncountable rank in finite semigroups of transformations and relations, Proc. Edinb. Math. Soc. 46, 531-544, 2003.

[11] J.M. Howie, Idempotent generators in finite full transformation semigroups, Proc. Roy. Soc. Edinburgh. Sect. A, 81 (3-4), 317-323, 1978.

[12] J.M. Howie, Fundamentals of Semigroup Theory, Oxford University Press, New York, USA, 1995.

[13] J.M. Howie and R.B. McFadden, Idempotent rank in finite full transformation semigroups, Proc. Roy. Soc. Edinburgh. Sect. A, 114 (3-4), 161-167, 1990.

[14] G.R. Ibrahim, A.T. Imam, A.D. Adeshola and G.N. Bakare, Some algebraic properties of order-preserving full contraction transformation semigroup, J. Semigroup Theory Appl. 2019 (2), 2019.

[15] K. Toker, Ranks of some subsemigroups of full contraction mappings on a finite chain (submitting).

[16] R.J. Wilson and J.J. Watkins, Graphs, An Introductory Approach, A First Course in Discrete Mathematics, Jon Wiley \& Sons Inc., Toronto, 1990.

[17] P. Zhao and V.H. Fernandes, The Ranks of ideals in various transformation monoids, Comm. Algebra, 43, 674-692, 2015. 